\title{
Composition anatomique de la carcasse du faisan de tir. Comparaison avec des poulets de poids similaires
}

\author{
F.H. Ricard ${ }^{1}$ et M.J. Petitjean 2 \\ avec la collaboration technique de $\mathrm{G}$. Marché
}

\begin{abstract}
1 INRA, station de recherches avicoles, Tours-Nouzilly, 37380 Monnaie;
2 INRA, laboratoire de spermiologie, domaine pluridisciplinaire du Magneraud, 17700 Surgères, France
\end{abstract}

(reçu le 23 novembre 1988, accepté le 28 février 1989)

\begin{abstract}
Résumé - Trois groupes de 31 faisans, deux de mâles et un de femelles, ont été élevés en volière pour être lâchés au moment de la chasse et ont été abattus selon les techniques du poulet de chair vers l'âge de 5 mois. Leur poids vif moyen était de $1426 \mathrm{~g}$. Ils ont été comparés à un lot de poulets abattus à l'âge de 7 semaines au poids vif moyen de $1501 \mathrm{~g}$.

Par rapport au poids vif, le pourcentage des dépôts gras abdominaux était en moyenne de 2,3\% chez le faisan (2,3\% également chez le poulet). La tête représentait $2,6 \%$ du poids vif chez le faisan $(2,9$ chez le poulet), les pattes $2 \%(4,4)$, les abats consommables $3,1 \%(4,1)$, le cou $3,1 \%(3,4)$, la carcasse éviscérée $71 \%(62)$, les muscles pectoraux $23 \%(12)$, les ailes $7 \%$ (8) et l'ensemble cuisses + pilons $23 \%(24)$. Le faisan de tir présente donc un très bon rendement à l'abattage et un remarquable développement des filets pour un étal d'engraissement comparable à celui du jeune poulet.

Les corrélations entre le poids vif et les éléments de la carcasse suggèrent qu'une augmentation de la vitesse de croissance risque de s'accompagner d'un plus fort pourcentage de gras abdominal et d'une diminution de l'importance des muscles pectoraux.
\end{abstract}

faisan - carcasse - gras abdominal - muscles pectoraux — rendements - corrélations

Summary - A comparison of the carcass compositions of game-type pheasants and broiler chickens with similar body weights. The carcass compositions of common pheasants (Phasianus colchicus) and domestic "broiler" chickens (Gallus gallus domesticus) with similar body weights were compared. The pheasants, two groups of 31 males and one group of 31 females, were reared in aviaries as if subsequently to be released for shooting. The pheasants were slaughtered when about 5 months old. At the time of slaughter the mean live body weight of the birds was $1426 \mathrm{~g}$. The broiler chickens were slaughtered when 7 weeks old at a live body weight of $1501 \mathrm{~g}$.

Expressed as a percentage of live body weight the carcass compositions of the pheasants and broiler chickens (shown in brackets) were as follows : abdominal fat deposits, $2.3 \%$ (2.3\%); head, $2.3 \%$ (2.9\%); shanks and feet, $2.0 \%$ (4.4\%); edible offal, 3.1\% (4.1\%); neck, 3.1\% (3.4\%); eviscerated carcass, $71 \%$ (62\%); pectoral muscles, $23 \%$ (12\%); wings, $7 \%(8 \%)$; thighs and legs, $23 \%$ (24\%). Thus, although the pheasants and broiler chickens exhibited similar levels of fattiness, the pheasants presented better slaughter yields and much greater breast meat percentages than the broiler chickens.

Phenotypic correlations indicated that improvement of growth rate in pheasants would lead to higher abdominal fat and lower pectoral muscles percentages.

pheasants - carcass composition - abdominal fat - pectoral muscles - yields - correlations 


\section{INTRODUCTION}

Le faisan (Phasianus colchicus L.) est un oiseau-gibier traditionnel dans de nombreuses régions du monde. En raison du développement de la chasse comme activité de loisir, de nombreux élevages se sont spécialisés en vue de produire des faisans "de tir» d'une manière aussi économique que possible. En France, des études scientifiques ont été entreprises pour préciser les conditions d'un élevage rationnel du faisan et un certain nombre de résultats ont été récemment présentés par Petitjean et al. (1986) ainsi que par Melin \& Larbier (1988). D'autres données sur les facteurs zootechniques de production, en particulier l'alimentation, peuvent être trouvées dans les travaux de Woodard et al. (1977), Warner et al. (1982), Dell'Orto et al. (1982) et Cain et al. (1984).

La commercialisation des carcasses de faisans, constituées au départ par les surplus de la chasse, prend maintenant de l'importance et entraîne un besoin de connaissances sur la composition anatomique de cette espèce. Or, peu de travaux, du moins à notre connaissance, ont été publiés. D'après Znaniecka (1973), la carcasse entièrement éviscérée représente environ $68 \%$ du poids vif. Pour Dell'Orto et al. (1982) la carcasse plumée représente $85 \%$ du poids vif. Le rendement en viande paraît être très élevé : pour Znaniecka (1973), l'ensemble des muscles de la poitrine et des membres inférieurs représente environ le tiers du poids vif et la moitié du poids de la carcasse éviscérée.

En vue de mieux connaître la composition anatomique des carcasses de faisans lâchés au moment de la chasse, plusieurs lots d'animaux de la souche expérimentale du Magneraud ont été abattus et disséqués dans notre laboratoire. Nous pré- sentons ici les résultats obtenus, en comparaison avec un échantillon de poulets de chair de poids vif comparable.

\section{MATÉRIEL ET MÉTHODES}

Les animaux provenaient de la souche expérimentale du Magneraud, mise en place en 1976 en vue d'étudier la faisabilité d'une reproduction en cage du faisan. La méthode d'élevage ainsi que quelques caractéristiques de développement corporel ont été présentées par Petitjean et al. (1986). Cette méthode d'élevage correspondait à la production d'oiseaux devant être lâchés pour la chasse à l'automne. II faut noter qu'à partir de l'âge de 5 semaines, les animaux (sexes mélangés) avaient accès à une volière ensemencée d'une flore complexe, avec une densité d'environ $3 \mathrm{~m}^{2}$ par sujet. Ce système d'élevage ne nous a pas permis d'estimer la consommation alimentaire des animaux.

Notre étude a porté sur 31 mâles et 31 femelles du cheptel né en juin 1978 et sur 31 mâles du cheptel né en juin 1979. Ils ont tous été pesés à l'âge de 18 semaines, en début de matinée mais sans avoir été mis à jeun. Ils ont été abattus ultérieurement, à l'âge de 158 jours (22 semaines et demi) pour les animaux nés en 1978 et à l'âge de 140 jours (20 semaines) pour ceux nés en 1979.

Les techniques utilisées étaient celles en usage dans notre laboratoire pour le poulet de chair. Les animaux ont été pesés juste avant l'abattage, après un jeûne complet d'environ $16 \mathrm{~h}$, saignés, échaudés et plumés à l'aide d'une machine à doigts de caoutchouc. Les carcasses ont ensuite été effilées, enveloppées dans du papier d'aluminium et placées en chambre froide $\left(+2\right.$ à $\left.+4^{\circ} \mathrm{C}\right)$ jusqu'au moment de la dissection qui avait lieu au cours de la semaine suivant l'abattage. Au cours de la dissection, les pattes ont été sectionnées au niveau de l'articulation tibiotarse-tarsométatarse et la tête à la jonction crâne-atlas. On a pesé séparément la tête, l'ensemble des deux pattes, les dépôts gras abdominaux (situés autour du ventricule succenturié, du gésier et contre la paroi abdominale), le gésier (sans la paroi interne cornée), le foie (sans la vésicule biliaire), le cour et le cou. Tous les autres viscères (œsophage, jabot, trachée-artère, pou- 
mons, rate, reins, gonades et glande de fabricius) ont été enlevés pour obtenir le poids de la carcasse éviscérée. Sur cette dernière, on a séparé et pesé l'ensemble des muscles pectoraux (c'est-à-dire les muscles situés à l'extérieur de la cage thoracique), les 2 ailes et l'ensemble cuisses + pilons. La dissection a été faite en suivant strictement les points de repère anatomiques, comme indiqué dans la brochure éditée par le Groupe de travail $n^{\circ} 5$ de la WPSA * (1984).

L'importance relative des différents éléments de la carcasse a été estimée en calculant les pourcentages par rapport au poids vif et par rapport au poids de la carcasse éviscérée. Les paramètres statistiques (moyenne, écart type) ont été calculés pour chaque variable puis les 3 groupes d'animaux (mâles 78 , femelles 78 , mâles 79 ) comparés à l'aide du test de Newmann \& Keuls (Dagnelie, 1975). Les corrélations phénotypiques faisant intervenir le poids vif avant abattage et les principaux rendements (gras abdominal, carcasse éviscérée, muscles pectoraux et ensemble cuisses + pilons) ont été calculées séparément pour chacun des 3 groupes de faisans, puis une valeur commune estimée après avoir transformé la corrélation en variable $z$, selon la technique décrite dans Dagnelie (1975).

Pour comparer les résultats observés chez le faisan à ceux qu'on peut trouver chez le poulet, nous avons repris les données publiées récemment (Ricard, 1988) concernant un lot de coquelets et de poulettes élevés en densité faible et abattus à l'âge de 7 semaines en 1983. Le choix de cet échantillon de poulets était motivé par le fait que les mêmes tech. niques d'abattage et de dissection avaient été utilisées et que les poids vifs à l'abattage étaient comparables, au moins dans le cas des mâles.

\section{RÉSULTATS ET DISCUSSIION}

Dans le Tableau I sont rassemblées les moyennes du poids vif et du poids des différents éléments de la carcasse, pour chacun des 3 groupes de faisans (mâles 78 , femelles 78 , mâles 79 ) et des 2 groupes de poulets (mâles 83 et femelles 83). Les écarts types indiqués correspondent à la racine carrée de la variance résiduelle obtenue au cours des analyses de variance correspondantes, valeurs également utilisées pour la comparaison des moyennes par le test de Newmann \& Keuls.

Les moyennes de poids vif font apparaître un dimorphisme sexuel nettement plus accentué chez le faisan (où le poids des femelles de notre échantillon représent moins de $70 \%$ du poids des mâles) que chez le poulet $(87,6 \%)$. Ce résultat concorde avec les chiffres indiqués par Stanhope (1974) où le rapport était de $71 \%$ chez des sujets âgés de 18 semaines, à ceux de Dell'Orto et al. (1982) où il approchait $73 \%$ pour des lignées de faisans de Mongolie et ceux de Petitjean et al. (1986) où il était de $72 \%$ pour des animaux âgés de 16 semaines appartenant à un autre échantillon de la même souche que celle étudiée ici. Les résultats présentés par Woodard et al. (1977) montrent que ce rapport diminue fortement quand l'âge augmente chez le faisan. En valeur absolue, les poids vifs de notre échantillon sont inférieurs à ceux cités par Stanhope (1974) pour une souche sélectionnée en Australie sur la vitesse de croissance. Ils sont par contre supérieurs aux valeurs observées sur les lignées de Dell'Orto et al. (1982) et sur les populations américaines étudiées par Woodard et al. (1977) ou par Cain et al. (1984).

Pour les animaux de notre échantillon nés en 1979 , le poids vif à 18 semaines est supérieur au poids mesuré à 20 semaines avant l'abattage. L'explication vient de ce que ce dernier était réalisé

\footnotetext{
* Groupe de travail mis en place par la Fédération européenne des Branches de la World Poultry Science Association.
} 
Tableau I. Caractéristiques de carcasse (valeurs brutes) mesurées dans 3 groupes de faisans et 2 groupes de poulets.

\begin{tabular}{|c|c|c|c|c|c|c|}
\hline \multicolumn{4}{|c|}{ Faisans ( $n=31$ par groupe) } & \multicolumn{3}{|c|}{ Poulets ( $n=20$ par groupe) } \\
\hline Mâles & Femelles & Mâles & Ecart type & Mâles & Femelles & Ecart \\
\hline 1978 & 1978 & 1979 & $\begin{array}{l}\text { intra- } \\
\text { groupe }\end{array}$ & 1983 & 1983 & $\begin{array}{l}\text { intra- } \\
\text { groupe }\end{array}$ \\
\hline
\end{tabular}

$\begin{array}{lrrrrrrr}\text { Poids vif 18 semaines } & 1576,1 \mathrm{a} & 1097,8 \mathrm{~b} & 1527,7 \mathrm{a} & 126,94 & - & - & - \\ \text { Poids vif avant abattage (1) } 1680,5 \mathrm{a} & 1144,8 \mathrm{c} & 1452,7 \mathrm{~b} & 136,96 & 1600,5^{\star} & 1401,9 & 119,35\end{array}$

$\begin{array}{lrrrrrrr}\text { Poids }(g) \text { des éléments de la carcasse } & & & & & & \\ \text { Dépôts gras abdominaux } & 46,8 \mathrm{a} & 31,3 \mathrm{~b} & 20,3 \mathrm{c} & 17,87 & 33,0 & 34,0 & 10,07 \\ \text { Tête } & 41,5 \mathrm{a} & 27,7 \mathrm{~b} & 41,9 \mathrm{a} & 2,50 & 47,9^{*} & 38,2 & 3,42 \\ \text { Pattes } & 33,6 \mathrm{a} & 22,3 \mathrm{~b} & 32,4 \mathrm{a} & 2,40 & 76,8^{*} & 57,4 & 6,13 \\ \text { Gésier } & 19,3 \mathrm{a} & 15,5 \mathrm{~b} & 19,2 \mathrm{a} & 4,84 & 28,0^{*} & 25,1 & 4,13 \\ \text { Foie } & 21,6 \mathrm{a} & 15,7 \mathrm{~b} & 21,3 \mathrm{a} & 2,22 & 29,8^{*} & 25,8 & 2,77 \\ \text { Cœur } & 7,9 \mathrm{a} & 4,6 \mathrm{~b} & 7,5 \mathrm{a} & 0,88 & 7,7^{*} & 6,2 & 0,92 \\ \text { Abats (gésier + foie + cœur) } & 48,7 \mathrm{a} & 35,8 \mathrm{~b} & 48,1 \mathrm{a} & 3,76 & 65,5^{*} & 57,0 & 5,82 \\ \text { Cou } & 52,3 \mathrm{a} & 32,0 \mathrm{c} & 48,7 \mathrm{~b} & 5,29 & 54,9^{*} & 48,4 & 5,50 \\ \text { Carcasse éviscérée } & 1199,7 \mathrm{a} & 812,9 \mathrm{c} & 1016,1 \mathrm{~b} & 105,53 & 995,5^{*} & 865,1 & 82,09 \\ \text { Muscles pectoraux } & 383,8 \mathrm{a} & 272,8 \mathrm{c} & 340,1 \mathrm{~b} & 26,33 & 196,1^{*} & 176,1 & 18,14 \\ \text { Ailes } & 107,9 \mathrm{a} & 75,6 \mathrm{c} & 100,5 \mathrm{~b} & 6,84 & 131,0^{*} & 112,4 & 10,78 \\ \text { Cuisses + pilons } & 398,0 \mathrm{a} & 265,7 \mathrm{c} & 341,4 \mathrm{~b} & 39,64 & 392,2^{*} & 325,8 & 32,58\end{array}$

(1) Age d'abattage : 22.5 semaines pour les faisans 78,20 semaines pour les faisans 79,7 semaines pour les poulets 83 .

a, b, c : Comparaison multiple des moyennes selon le test de Newmann et Keuls. Deux valeurs suivies de la même lettre ne sont pas statistiquement différentes $(P>0,05)$. La lettre a correspond à la valeur la plus élevée.

* Différence significative $(P<0,05)$ entre poulets mâles et poulets femelles.

après un jeûne complet de $16 \mathrm{~h}$, ce qui entraîne une perte de poids supérieure au gain réalisé entre 16 et 18 semaines pour des animaux non à jeun. D'autre part, les mâles abattus en 1978 étaient significativement plus lourds $(228 \mathrm{~g})$ et avaient deux fois plus de dépôts gras abdominaux $(46,8$ contre $20,3 \mathrm{~g})$ alors qu'il s'agit de la même souche d'animaux. Une explication possible est la différence d'âge (18 jours) et de poids au moment de l'abattage. Nous n'avons pas de données sur l'évolution du poids vif et de l'engraissement en fonction de l'âge sur des faisans de la même souche, mais d'après les chiffres cités par Woodard et al. (1977) cette explication est insuffisante. Une autre cause de variation pourrait être un effet année lié aux conditions climatiques durant l'élevage, ce qui peut jouer à la fois sur la végétation de la volière et sur la physiologie des animaux : une sécheresse peut réduire le couvert végétal et une augmentation de la température peut diminuer la croissance juvénile du faisan (Warner et al., 1982). Malheureusement, les conditions climatiques n'ont pas été relevées au cours de l'expérimentation. II n'est donc pas possible de discuter ce point plus avant. II est à noter que les 
Tableau II. Rendements, exprimés en pourcentages, par groupe de faisans et de poulets.

\begin{tabular}{|c|c|c|c|c|c|c|c|}
\hline & \multicolumn{4}{|c|}{ Faisans ( $n=31$ par groupe) } & \multicolumn{3}{|c|}{ Poulets ( $n=20$ par groupe) } \\
\hline & $\begin{array}{l}\text { Mâles } \\
1978\end{array}$ & $\begin{array}{l}\text { Femelles } \\
1978\end{array}$ & $\begin{array}{l}\text { Mâles } \\
1979\end{array}$ & Ecart type & $\begin{array}{l}\text { Mâles } \\
1983\end{array}$ & $\begin{array}{l}\text { Femelles } \\
1983\end{array}$ & $\begin{array}{l}\text { Ecart } \\
\text { type }\end{array}$ \\
\hline \multicolumn{8}{|l|}{ En \% du poids vif } \\
\hline Dépôts gras abdominaux & $2,71 \mathrm{a}$ & $2,67 a$ & $1,35 b$ & 1.08 & $2,04^{*}$ & 2,42 & 0,59 \\
\hline Tête & $2,49 b$ & $2,43 b$ & $2,90 a$ & 0,23 & $3,00^{*}$ & 2,73 & 0,14 \\
\hline Pattes & $2,01 b$ & $1,95 b$ & $2,24 a$ & 0,14 & $4,80^{*}$ & 4,09 & 0,23 \\
\hline Gésier & $1,16 b$ & $1,36 a$ & $1,33 a$ & 0,18 & 1,76 & 1,79 & 0,28 \\
\hline Foie & $1,30 \mathrm{~b}$ & $1,38 b$ & $1,48 a$ & 0,17 & 1,86 & 1,84 & 0,15 \\
\hline Coeur & $0,47 b$ & $0,41 \mathrm{c}$ & $0,52 a$ & 0,06 & $0,48^{*}$ & 0,44 & 0,05 \\
\hline Abats consommables & $2,93 c$ & $3,15 b$ & $3,32 a$ & 0,31 & 4,10 & 4,07 & 0,34 \\
\hline $\mathrm{Cou}$ & $3,13 b$ & $2,79 c$ & $3,36 a$ & 0,33 & 3,44 & 3,45 & 0,29 \\
\hline Carcasse éviscérée & $71,34 a$ & $70,95 a$ & $69,71 b$ & 1,28 & 62,15 & 61,70 & 1,20 \\
\hline Muscles pectoraux & $22,95 \mathrm{~b}$ & $23,90 a$ & $23,45 a$ & 1,57 & 12,26 & 12,57 & 0,76 \\
\hline Ailes & $6,44 b$ & $6,63 b$ & $6,94 a$ & 0,38 & 8,19 & 8,02 & 0,28 \\
\hline Cuisses + pilons & $23,65 a$ & $23,14 a$ & $23,49 a$ & 0,98 & 24,49 * & 23,23 & 0,68 \\
\hline \multicolumn{8}{|c|}{ En \% du poids de la carcasse éviscérée } \\
\hline Muscles pectoraux & $32,18 b$ & $33,69 a$ & $33,55 a$ & 2,24 & $19,71(1)$ & 20,36 & 1,06 \\
\hline Ailes & $9,04 c$ & $9,35 \mathrm{~b}$ & $9,93 a$ & 0,60 & 13,18 & 13,00 & 0,42 \\
\hline Cuisses + pilons & $33,14 a b$ & $32,61 \mathrm{~b}$ & $33,60 a$ & 1,22 & $39,40^{*}$ & 37,65 & 0,77 \\
\hline
\end{tabular}

a, b, c, * : voir Tableau I.

(1) différence proche du seuil de signification $5 \%$.

poids des abats (tête, pattes, gésier, foie, cœur) ne sont pas significativement différents pour les mâles abattus en 78 et en 79.

Les rendements, exprimés en pourcentages du poids vif et du poids de la carcasse éviscérée sont regroupés dans le Tableau II. Contrairement à ce qui se passe chez le poulet, le pourcentage des dépôts gras abdominaux est identique pour les 2 sexes dans notre échantillon de faisans. II serait intéressant de voir si cela correspond à une même teneur en lipides de la carcasse ou s'il s'agit d'un résultat particulier à notre échantillon. Mais cela demanderait une expérimentation complémentaire. L'importance des abats est plus faible chez le faisan de tir que chez le poulet de chair, en particulier en ce qui concerne les pattes, le gésier, le foie et le cou. II en résulte un pourcentage de carcasse éviscérée nettement plus élevé chez le faisan (de l'ordre de $71 \%$ ) que chez le poulet (62\%). Ce pourcentage est significativement plus élevé pour les mâles 78 que pour les mâles 79 , mais nous n'observons pas de différence significative entre les 2 sexes en 1978.

Comme chez le poulet, les femelles faisanes ont tendance à avoir des muscles pectoraux plus développés que les mâles et présentent un pourcentage de cuisses et pilons un peu moins élevé (et non significativement différent dans notre échantillon). Les muscles pectoraux et les ailes sont significativement plus 
développés chez les mâles abattus en 1979 que chez ceux abattus en 1978. La comparaison des espèces faisan et poulet met en lumière une importante différence dans la répartition des parties de carcasse : les muscles pectoraux sont nettement plus développés chez le faisan (près du quart du poids vif et un tiers du poids éviscéré) que chez le poulet (12\% du poids vif et $20 \%$ du poids éviscéré). En contrepartie, les membres (ailes, cuisses et pilons) représentent une part plus faible chez le faisan que chez le poulet. On peut également remarquer que les muscles pectoraux et l'ensemble cuisses + pilons représentent le même pourcentage de la carcasse chez le faisan, alors que chez le poulet l'ensemble cuisses + pilons représente près du double des muscles pectoraux. Ces résultats confirment les données de Znaniecka (1973) concernant le rendement en viande élevé des carcasses de faisan.

Les valeurs des corrélations phénotypiques entre le poids vif et les principaux rendements (dépôts gras abdominaux, muscles pectoraux, ensemble cuisses + pilons) sont indiquées dans le Tableau III. Le poids vif avant abattage est plus fortement corrélé avec le poids des parties de carcasse qu'avec les pourcentages, ce qui est logique. On peut constater que la liaison entre poids vif et engraissement (poids ou pourcentage du gras abdominal) est plus forte chez le faisan que chez le poulet. Le pourcentage de muscles

Tableau III. Corrélations phénotypiques entre le poids vif avant abattage, et les principaux rendements de la carcasse chez le faisan et le poulet.

\begin{tabular}{|c|c|c|c|c|c|}
\hline \multirow[b]{2}{*}{ Caractères corrélés } & \multirow[b]{2}{*}{$\begin{array}{c}\text { Mâles } \\
1978\end{array}$} & \multicolumn{2}{|c|}{ Faisans (1) } & \multirow[b]{2}{*}{$\begin{array}{l}\text { Valeur } \\
\text { commune }\end{array}$} & \multirow[t]{2}{*}{ Poulets 83 (2) } \\
\hline & & $\begin{array}{l}\text { Femelles } \\
1978\end{array}$ & $\begin{array}{l}\text { Mâles } \\
1979\end{array}$ & & \\
\hline \multicolumn{6}{|l|}{ Poids vif avant abattage et: } \\
\hline Poids vif 18 semaines & 0,92 & 0,92 & 0,96 & 0,936 & - \\
\hline Poids gras abdominal & 0,82 & 0,65 & 0,75 & 0,750 & 0,542 \\
\hline Poids carcasse éviscérée & 0,99 & 0,98 & 0,98 & 0,987 & 0,977 \\
\hline Poids muscles pectoraux & 0,75 & 0,60 & 0,87 & 0,760 & 0,759 \\
\hline Poids cuisses + pilons & 0,96 & 0,93 & 0,96 & 0,951 & 0,947 \\
\hline$\%$ gras abdominal/poids vif & 0,72 & 0,57 & 0,70 & 0,668 & 0,344 \\
\hline \% carcasse éviscérée/poids vif & 0,47 & 0,41 & 0,29 & 0,393 & $0,285 \mathrm{NS}$ \\
\hline$\%$ muscles pectoraux/éviscéré & $-0,76$ & $-0,51$ & $-0,56$ & $-0,624$ & $-0,207 \mathrm{NS}$ \\
\hline$\%$ cuisses + pilons/éviscéré & 0,28 & 0,50 & 0,00 & 0,275 & $-0,014 N S$ \\
\hline \multicolumn{6}{|l|}{$\%$ gras abdominal/poids vif et : } \\
\hline$\%$ muscles pectoraux/éviscéré & $-0,80$ & $-0,83$ & $-0,53$ & (3) & $-0,416$ \\
\hline$\%$ cuisses + pilons/éviscéré & 0,33 & 0,69 & 0,01 & (3) & $0,108 \mathrm{NS}$ \\
\hline \multicolumn{6}{|l|}{$\%$ muscles pectorauX'éviscéré et : } \\
\hline$\%$ cuisses + pilons/éviscéré & $-0,55$ & $-0,79$ & $-0,56$ & $-0,650$ & $-0,327$ \\
\hline
\end{tabular}

(1) Pour chaque groupe de faisans, le seuil de signification à $5 \%$ de la corrélation est de 0,35 .

(2) Valeur commune aux poulets mâles et femelles.

(3) Valeur commune non indiquée par suite de l'hétérogénéité des corrélations pour les 3 groupes de faisans.

NS = valeur commune non significativement différente de zéro $(P>0,05)$. 
pectoraux est corrélé négativement avec le poids vif, assez fortement chez le faisan et de façon non significative dans notre échantillon de poulets. La corrélation entre poids vif et pourcentage des cuisses et pilons n'est significative que pour le groupe de faisans femelles. Le pourcentage de dépôts gras abdominaux est corrélé négativement avec le pourcentage de muscles pectoraux, plus fortement chez le faisan que chez le poulet. Entre pourcentages de gras abdominal et de cuisses et pilons, la corrélation est positive pour les faisans du cheptel 78 mais pratiquement nulle pour ceux du cheptel 79. Enfin, les pourcentages de muscles pectoraux et de cuisses et pilons sont corrélés négativement, de façon plus forte chez le faisan que chez le poulet. Ce résultat est la conséquence "mécanique" du fait que ces 2 éléments représentent la plus grande partie de la carcasse éviscérée et que si la part des muscles pectoraux augmente, celle des cuisses et pilons doit logiquement diminuer.

Dans l'ensemble, les corrélations présentées dans le Tableau III permettent de dire que, chez le faisan, l'augmentation du poids vif s'accompagne d'un engraissement plus élevé et d'une diminution de l'importance des muscles pectoraux. Chez le poulet, la même tendance existe mais de façon beaucoup plus modérée.

\section{CONCLUSION}

On peut dire que le faisan peut constituer une excellente volaille de boucherie : le rendement à l'abattage est élevé, l'engraissement ne paraît pas excessif, le développement des filets est tout à fait remarquable. Mais on n'a sans doute pas intérêt à rechercher un poids vif trop élevé si l'on veut conserver ces caractéristiques. Par ailleurs, il serait intéressant d'avoir des données comparables sur d'autres types de souches pour voir si nos résultats sont généralisables, et sur des animaux abattus à des âges différents pour étudier l'évolution de la composition anatomique du faisan en fonction de l'âge. Enfin, il est certain que si le faisan devenait une volaille de boucherie, il faudrait développer des études sur les conditions d'élevage spécifiques à ce type de production.

\section{RÉFÉRENCES}

Gain J.R., Weber J.M., Lockamy T.A. \& Creger C.R. (1984) Grower diets and bird density effects on growth and cannibalism in ring-necked pheasants. Poult. Sci. 63, 450-457

Dagnelie P. (1975) Théorie et Méthodes Statistiques. Vol. 2 : Les méthodes de l'inférence statistique, Presses Agronomiques, Gembloux (Belgique), pp. 341-358

Dell'Orto V., Corino C., Guidoboni G. \& Zerbinatti L. (1982) Effetti dell'impiezo di differenti livelli proteici nella dieta del fagiano in crescita. Riv. Avic. 51, 25-30

Melin J.M. \& Larbier M. (1988) Influence du taux protidique de l'aliment de démarrage sur les performances de croissance et d'emplumement du faisan. Ann. Zootech. 37, 143-150

Petitjean M., Guillot P. \& Malineau G. (1986) Maîtrise de la reproduction du faisan. Comples Rendus 7e Conf. Europ. Avic. (août 1986, Paris, Larbier M., éd.). Vol. 1, pp. 148-152

Ricard F.H. (1988) Influence de la densité d'élevage sur la croissance et les caractéristiques de carcasse de poulets élevés au sol. Ann. Zootech. 37, 87-98

Stanhope W. (1974) Commercial pheasant production. Proc. Australasian Poult. Sci. Conv. Hobart, Australian branch WPSA ed. pp. 282286 
Warner R.E., Darda D.M. \& Baker D.H. (1982) Effects of dietary protein level and environmental temperature stress on growth of young ringnecked pheasants. Poult. Sci. 61, 673-676

Woodard A.E., Wohra P. \& Snyder R.L. (1977) Effect of protein levels in the diet on the growth of pheasants. Poult. Sci. 56, 1492-1500
WPSA, Working group 5 (1984) Method of Dissection of Broiler Carcasses and Description of Parts (Jensen J.F., ed.) Pergamon Press, Cambridge (UK)

Znaniecka H. (1973) Produktionsmöglichkeiten von mastfasanen (en polonais). Zesz. Nauk. Akad. Roln. Techn. Olsztynie, Zootec. 2, 153157 